\title{
Educational Scientific Research is the Core Power of Teachers' Professional Development
}

\author{
Qiduan Chen ${ }^{1, a}$, Qingqing Zhang ${ }^{2, b^{*}}$ \\ ${ }^{1}$ JiMei University, Jimei, Xiamen, Fujian, China \\ 2JiMei University, Jimei, Xiamen, Fujian, China \\ 1140751800@qq.com \\ b*2435705947@gq.com
}

\section{ABSTRACT}

Scientific research is one of the meaning of specialized occupation, is the lifeline for the development of specialized occupation, is one of the sustained and healthy development of the special features, marks an important and irreplaceable to maintain professional activities. Therefore, the structure of teachers' professional quality in theory should be a four-dimensional model of Pyramid, including five aspects of teachers' educational scientific research accomplishment, professional theory, professional knowledge, professional skills and professional ethics. Among them, education scientific research qualities is the core power of teachers professional development; teachers continuously improve their professional level "energy bar"; is the structure of teachers' professional quality kernel, living in the center of the structure of teachers' professional quality. It is through the system overlap and cross influences and the quality of the work of other systems working state.

Keywords: Teacher scientific literacy, Education specialty quality, Status, Model

\section{教育科学研究是教师专业发展的核心动力}

\author{
陈其端 ${ }^{1, a}$ ，张青青 ${ }^{2}$, b*
}

\footnotetext{
${ }^{1}$ 集美大学, 集美, 厦门, 福建, 中国

集美大学, 集美, 厦门, 福建, 中国

a 1140751800@qq.com

b*2435705947@qq.com
}

\section{摘要}

教师的专业素质结构在其理论上应该是一个四维的金字塔模型，包括教师的教育科学研究素养、专业理念、专 业知识、专业能力和专业伦理五个方面。其中, 教师专业发展的核心素质就是教育科学研究素养; 教育科学研 究素质是教师在教育教学过程中不断提升自身专业水平的 “能量棒” ; 更是教师专业素质结构的内核, 居于教 师专业素质结构的中心地位。教育科学研究素质通过系统间的相互作用并且影响着其他系统的工作状态和工作 质量。

关键词: 教育科学研究素养; 教师专业素质; 地位; 模型

\section{1. 前言}

教师专业素质, 在国内较为权威的解释是指: “教 师从事教学所必备的专门知识, 技能, 以及为完成教 育教学任务所应具备的心理和行为品质的基本条件, 教师的专业素质主要包括扎实的理论基础、熟练的专
业技能、全面的业务能力三个方面”。 ${ }^{[1]}$. 显然, 它是 对教师专业 “底线” 的基本要求。然而在日常生活中, 人们则更关注教师专业素质结构的问题。教师专业素 质究竟包含哪些内容 (维度), 国内学者研究颇丰 (可 见下文表格），有学者通过综述性研究指出：“尽管 目前学术界关于教师专业素质内容结构依然没有较 
为准确的解释, 但是相当一部分的研究表明：专业知 识、专业技能、专业情意是现代教师专业素质集中体 现的三个代表性特征” ${ }^{[2]}$ 也有部分学者通过研究补充 认为：“尽管国内外相关研究对教师所必须具备的 素质各有不同的看法, 但通过分析和综合比对, 关于 教师素质的认识可以被概括的划分为五个方面的内 容, 即: 职业道德、教育理念、知识、能力、教师的个 性品质或人格特质。” ${ }^{[3]}$

然而, 我们通过研究了解到无论是理论知识方面 的研究还是实际生活中的要求, 教师专业素质结构在 维度上都缺乏 “教育科学研究素养” 这一对于专门职 业而言非常重要的素质结构维度。接下来笔者就教育 科学研究素养在教师专业素质结构中的核心地位及 其现实落差, 以及教育科学研究在教师专业发展中的 核心动力模型构想等问题做一些简单的探讨, 以期方 家之教。

\section{2. 教育科学研究素养在教师专业素质结构中 的核心地位}

与上述关于教师专业素质的权威解释相比, 笔者 更同意一种观点: “ “教师专业素质” 是指教师经过 系统的专业化学习, 并在长期的教学实践中逐渐发展 而成的具有专门性、指向性和不可替代性的素质, 强 调的是教师职业的 “独特性”、“标志性” ”。每可 见, 教师专业素质不同于教师素质。教师素质 “指教 师所应该具备的各种综合素质，体现的是追求教师的 理想化形象, 突出教师的 “全面性、理想性” ” ; ${ }^{[5]}$ 教师专业素质指的是教师这一专业所具有的专门性、 指向性和不可替代性的内在特质。研究表明, 科研素 质可以认为是体现教师专业的特殊性、标志性和不可 替代性的重要特征之一。换句话说, 一种专门职业是 需要不断提高其专业化程度来巩固其社会地位而不 至于沦落为普通职业的, 而教师专业化程度的提高大 部分原因要归结于教师在教学和科研活动中所做出 的成就。因此可以得出, 教师专业素质结构的中心位 置非教师科研素养莫属。

\section{1. 科学研究是专门职业发展的核心动力}

有学者指出: “人类的活动可以大约分成三个不 同的层次: 本能水平、经验水平和有意识的反思水平。 维持我们生存的基本活动称之为本能活动, 它属于种 族遗传性质; 经验活动是人类从古至今将自己代代相 传的活动经验积累起来, 属于原始模仿性质; 而有意 识的反思则是将活动的主体和客体分离开来, 对活动 的特点、过程和规律进行理性的分析, 属于研究性 质。” ${ }^{[6]}$ 根据这一认识, 我们发现, 在人类现实的社 会活动中, 如果以个体活动中某项活动水平占有绝对 比重而言, 这三种人类活动的水平可对应于三种职业 类型, 即无业、普通职业和专门职业（简称专业）。

比如说, 一个人的本能性活动在其活动中占有绝 对比重, 以吃、喝、拉、撒等本体性需求的满足为主
要活动目标, 他在社会职业方面可能性最大的是无业 的, 这一类活动水平的特点是一般不具有反思性。按 照马斯洛需求层次理论, 职业需求是人的自我实现等 高层次需求之一, 而以满足机体本身需要的无业状态 的需求恰恰是人类最低层的生理需求。

而经验性活动主要指的是个体需要从多次实践 中获取所从事活动的知识和技能, 注重个体的亲身经 历性。这一类水平的活动对应于社会职业结构中的普 通职业, 其特点是注重个人实际操作技能的反复练 习, 很少对于从事的活动表现出一定的反思性, 甚至 在主观感受中不喜欢反思或者惧怕反思, 因为反思的 结果很可能会消灭一个职业。比如说, 我国 20 世纪 的公共汽车上都配备有专门的售票人员, 但是随着车 载自助投币机的发明, 这类售票员的职业基本消失。 具有戏剧性的是, 公共汽车自助投币机的发明初衷之 一就是为了分担售票员的繁忙劳动, 让售票工作更加 有效。类似的案列在历史上数不胜数, 相似的结果往 往是机器的出现会慢慢吞噬掉某个普通职业, 职业工 人面临下岗的宭境。因此, 普通职业者是很少进行反 思的, 或者说反思性活动不是这类职业的内涵。

与上述二者不同的是, 人类有意识的反思性活动 水平具有极强的研究性特征, 对应的职业类型就是专 门职业。专门职业天生具有研究的特性, 反思性研究 活动是专业的内在维度。举例来说, 医生是一种专门 职业, 医疗器械和医药的大量发明是基于医务专业人 才的研究与创造, 而这些研究成果的大量应用又成功 地推动医务工作者专业技能的提升和医疗水平的大 幅提高, 并未因为医学方面新的发明和创造使得医生

下岗或者医生职业消失, 研究水平的高低已然成 为医生执业水平高低的晴雨表。其他高度专业化的职 业

亦是如此, 不再赘述。

综上所述, 对于专门职业而言, 科学研究成为这 类职业的生内在动力。科学研究的问题来自于专业实 践, 科学研究的成果又直接应用于专业实践, 并且提 高其专业化水平, 最终巩固了专门职业的社会地位和 入职门槛。相反, 如果一个专业不重视有意识的反思 性活动, 即忽视科学研究, 将直接影响到这一专业的 服务水平和社会地位, 最终会导致其睢落成不以反思 性活动为内涵的普通职业。因此, 科学研究素养是专 门职业发展的核心动力, 是专门职业赖以发展的生命 线, 是保持专业活动持续健康发展的特殊性和不可替 代性的重要特征之一, 即可以理解为科学研究素养是 各种专业素质结构中的内核。

\section{2 教育科学研究是教师专业发展的核心动 力}

目前, 关于教师是一门专业在国际上已达成共 识。在中国, 受到孔子 “三人行必有我师” 思想影响 较为广泛和深远的民间还有 “谁都可以当老师” 的认 识。虽然流传这样的说法, 一些法律和政策还是清晰 地指出 “教师是从事教育教学的专门人员”。教师是 
一门专业, 哪怕至今为止教师的专业化程度没有想象 中的那么高, 但是作为一门专业就应该有那些高度专 业化的职业, 即专业的不可替代性, 比如 “医生不是 任何人随便做的”已成为普遍共识。正如前所述, 这 一反映专业特殊性的共识恰恰是由医生专业的科研 素质内核来提升和巩固的。

而教师专业要想成为 “不是任何人随便做教师” 的专门职业, 就应该认识到专门职业是人类活动水平 的最高层次, 教师职业作为专门职业亦是人类 “有意 识的反思性活动”之一, 研究是这一类活动水平的核 心所在。因此, 教育科学研究活动不是外在力量强加 给老师的工作负担, 而是教师的专业素质结构中的重 要内核。那种以工作忙没有时间搞研究等为借口逃避 反思性研究活动的教师, 实际上还是停留于 “教书匠” 的普通职业水平。

当然, 我们也不赞同那种 “没有科研的教学是低 水平的教学” 的论点, 因为作为 “教书匠” 式的论为 “普通职业的教师” 的教学并不见得是低水平的, 就 如作为临时职业的清洁工清扫地面一样, 并不因为从 事的是 “经验性活动” , 他就比普通或者专门工作者 清扫地面差到哪里去。一名教师只要他有足够的学科 背景, 加上较强的职业道德和职业精神, “教书匠” 依然可以成为 “教书将”。我们经常发现大、中、小 学许多教学名师, 也许一辈子不做科研活动, 他依旧 教学很出色。但是, 我们说这些名师的成功道路是曲 折而艰辛的, 是靠一次次的经验性的实践性知识的积 累最终才取得的成就, 这恰恰就是人类“经验性活动” 水平的明显特征, 即 “个体经历性” 的最好注脚。因 此, 作为普通职业的 “教书匠” 或者 “教书将” , 他 的经验性教育教学具有明显的个体性、积累性和盲目 性的特征。

经过上述分析, 教师的专业化, 无论是群体性专 业化还是个体性专业化显然绝对不能依靠 “经验性活 动水平” 来推进, 要想教师成为 “谁都不可以随便当” 的专门职业, 就要认识到教师专业素质结构中存在的 教育科学研究素养内核, 它是探讨教师专业素质结构 维度时不可或缺的重要内容和原生动力。然而, 我们 发现, 在已有理论研究和我国教师标准的实际要求 中，对于教师科研素质的重视还远远不够。

\section{3. 教育科学研究素养在教师专业素质结构中的地 位落差}

下面, 笔者就目前国内学界在教师专业素质结构 的研究中, 以及在我国已颁布施行的教师标准中, 关 于教育科学研究素养缺乏关注的现状, 即教育科学研 究素养的地位落差做一探讨, 借此来反映在当前提高 教育科研素养核心在教师专业素质结构中是必要的。

\section{1. 教育科学研究素养在教师专业素质研究 中的地位落差}

学术界对于教师教育科学研究素养能力这方面 的知识已经做研究, 但对于教育科学研究素养的定位 尚有空白。笔者通过研究发现, 学界对于教师专业素
质结构的研究具有一定影响的观点如下表所示:

表：教师专业素质结构研究

\begin{tabular}{|c|c|}
\hline 研究者 & 教师专业素质结构的基本观点 \\
\hline 叶澜 ${ }^{[1]}$ & 1、专业理念; 2 、知识结构; 3 、能力结构 \\
\hline 林瑞钦 ${ }^{[2]}$ & $\begin{array}{l}\text { 1、所教学科的知识（能教）; 2、教育专 } \\
\text { 业的知能（会教）; 3、教育专业精神（愿 } \\
\text { 教） }\end{array}$ \\
\hline 姚念章 ${ }^{[3]}$ & 1、认识系统; 2、情意系统; 3、操作系统 \\
\hline 唐松林 ${ }^{[4]}$ & 1、认知结构; 2、专业精神; 3、教育能力 \\
\hline 艾伦 & 1、学科知识; 2 、行为技能; 3 、人格技能 \\
\hline 饶见维 ${ }^{[6]}$ & $\begin{array}{l}\text { 1、教师通用知能; 2、学科技能; 3、教育 } \\
\text { 专业知能; 4、教育专业精神 }\end{array}$ \\
\hline 孟万金 $^{[7]}$ & $\begin{array}{l}\text { 1、专业理念系统；2、专业智能系统；3、 } \\
\text { 专业情怀系统； 4、专业规范系统 }\end{array}$ \\
\hline 谢安邦 ${ }^{[8]}$ & $\begin{array}{l}\text { 1、教育理念结构; 2、职业道德结构; } 3 \text { 、 } \\
\text { 知识结构; 4、能力结构 }\end{array}$ \\
\hline 林崇德 ${ }^{[9]}$ & $\begin{array}{l}\text { 1、教师职业理想; } 2 \text { 、知识水平; } 3 \text { 、教育 } \\
\text { 观念 } ; 4 \text { 、教学监控能力; } 5 \text { 、教学行为策 } \\
\text { 略 }\end{array}$ \\
\hline
\end{tabular}

综观上述观点, 教育科学研究素养并没有被研究 者列在教师专业素质结构维度之中。它更多地只是作 为教师的一种能力而存在于教师专业能力结构的讨 论中, 如有研究者指出: “能力是个体的一种心理特 征, 不仅为个体完成活动提供一定的可能性, 而且影 响活动效率。众所周知一个优秀的教师, 不仅是要成 为学生学习的促进者、社会改革者和反思型实践者, 同时还需要具备六种基本能力: 了解学生的能力、教 育教学的能力、交际能力、反思能力、教育研究能力、 学习能力。” ${ }^{[7]}$ 然而, 笔者认为上述研究者指出的 “教 育研究能力” 并非教师教育科学研究素养的全部, 单 纯强调教育研究能力, 却忽视了教育科学研究素养的 其它构成要素, 进而弱化教师科学研究素养在教师专 业素质结构中的地位。要知道, 教育科学研究素养归 为科学研究素质中的一方面。“科研素质是指研究个 
体的学术活动的过程, 在科研道德品性、科研知识和 科研能力等方面形成的固有、相对恒定的科研内在条 件。” ${ }^{[8]}$ 由此可见, 教育科学研究素养是教师专业的 一种内在需求, 它在内部结构上至少应该包括: 教育 科学研究素养知识、教育科学研究素养能力、教育科 学研究素养精神和教育科学研究素养道德等方面。教 育科学研究素养有着自身完整的构成, 缺一不可, 整 体上对于教师从事教育教学研究和其它教育专业活 动发挥着重要作用。因此, 以往研究单纯将教育研究 能力从教育科学研究素养中抽离出来作为教师专业 能力的一种进行讨论, 这在理论上, 使得教育科学研 究素养内涵在诠释和评价方面呈现片面化特征, 从而 降低了教育科学研究素养的重要地位。

\section{2 教育科学研究素养在教师专业标准维度中 的地位落差}

在实际规定中，2012 年 2 月 10 日，教育部下发 了关于《幼儿园教师专业标准 (试行) 》《小学教师 专业标准 (试行) 》和《中学教师专业标准 (试行)》 的通知, 我们可以看出在教师标准的相关政策文本 中, 教育科学研究素养尚未与教师的 “专业理念与师 德”、“专业知识” 和 “专业能力” 并列成为教师的 专业素质维度。

幼儿园、中小学教师标准的文本中只是将 “反思 与发展” 作为一种专业能力做出粗略的规定要求: 1 、 教师要积极主动去收集、思考并整合相关信息, 进行 深刻反思, 从而不断提高和丰富教育教学的工作水 平; 2、针对教育教学工作中的现实需要与问题, 进 行深入探索和研究; 3、制定出专业发展的规划, 主 动参加教师相关培训, 逐渐提高自身的专业素质。

中等职业学校教师标准的文本中关于教育科学 研究素养方面的要求相比幼儿园、中小学而言更高, 将 “反思与发展” 能力换成 “教学研究与专业发展” 能力, 具体要求有: 1、主动收集分析毕业生毕业后 的就业情况和用人单位的用人需求等相关信息, 不断 反思和改进教育教学工作; 2、针对教育教学工作中 的现实需要与问题, 进行探索和研究; 3、参加校本 教学研究和教学改革; 4、结合行业企业需求和专业 发展需要, 制定个人专业发展规划, 通过参加专业培 训和企业实践等多种途径，不断提高自身专业素质。

解读上述教师标准中对于教师科研素质方面的 要求, 我们发现, 教育科学研究素养在现实政策文本 要求中存在着片面化的问题是显而易见的。首先, 教 师标准在制定理念上缺乏对幼儿园、中小学甚至中等 职业学校教师从事教育科学研究活动的充分认可与 自信。标准中用 “反思与发展” 和 “教学研究与发展” 等片面化的 “能力” 术语, 而非 “教育科学研究素养” 这一更宽范畴的上位概念, 恰恰说明教育界对于中小 学教师从事正规教育科学研究活动的不确定性或者 较强的不信任感; 其次, 教师标准在具体要求方面, 用 “反思与发展” 或 “教学研究与发展” 的片面性理 解置换了教育科学研究素养的全部内涵, 缺乏对于教 育科学研究素养意识、科研精神和科研道德等重要内
涵足够的认识和要求, 使得教育科学研究素养这一教 师专业素质的内在要求堕落成为对教师的一种外在 的能力要求, 这直接造成诸多教师认为教育科学研究 素养是教育主管部门对教师工作的外在要求的主观 感受; 最后, 教师标准在评价方面, 因只注重 “反思 与发展” 或者 “教学研究与发展” 能力方面指标的终 结性评价, 如发表教学研究论文的数量等, 而缺乏对 于教师在教育教学研究中表现出的生动的科研意识、 科研精神以及科研道德等的生成性评价或者过程性 评价, 教师的教育研究因此流于表面和形式, 因此并 不能从真正意义上推动教师的专业化发展。

实际上, 因为教师专业素质结构在研究或规定中 缺乏教育科学研究素养这一重要维度, 已经带来诸多 实际问题。例如, 非常突出的问题是: 现在中小学教 师大都缺乏教育科学研究素养意识, 对教育科学研究 素养是教师专业的重要内涵没有深刻的认识, 教育科 学研究素养是从事教师专业活动的内在需求, 从而将 教育研究当成可有可无的外部事情, 甚至当成影响和 占用教学工作的绊脚石, 普遍对于教育科学研究素养 带有较强的抵触或者畏难情绪; 又如, 由于管理上只 强调和衡量教师教育科学研究素养的能力或教育教 学反思能力, 忽视教育科学研究素养道德与科研精神 内涵的要求和养成, 科研工作中抄袭和剽窃现象屡禁 不止, 成为当前教育科学研究素养工作的顽疾。由此 得出, 教育科学研究素养应该成为教师专业素质的重 要维度和重要内涵。

\section{3. 教育科学研究在教师专业发展中的核心 动力模型}

在以往研究基础上, 近年来的相关研究似乎达成 了一个共识, 即认为教师专业素质结构存在一个三维 结构。如李建辉、王晶晶的《教师专业素质结构新探》 一文指出: “教师专业素质的结构体系主要包括专业 理念、专业知识、专业能力和专业规范四个方面, 其 理论结构形态如下模型图所示: ${ }^{[9]}$

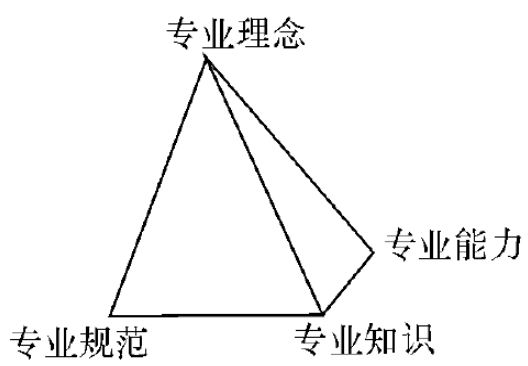

图 1：教师专业素质结构体系三维图（笔者注）

而苏志刚的《论高职院校教师专业素质三维结 构》 ${ }^{[10]}$ 一文更将此三维结构理论模型精细化为如下图 所示: 


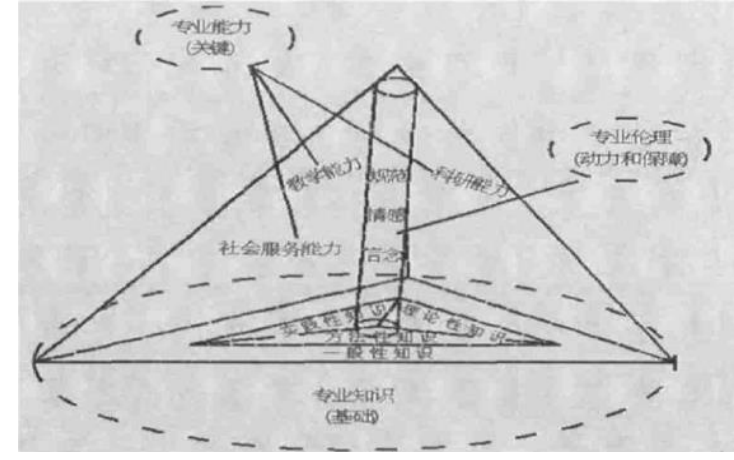

上述教师专业素质三维结构的研究为我们探讨 教育科学研究素养回归教师专业素质结构提供了新 的视野。然而, 基于前文的阐述, 我们认为在上述教 师专业素质结构的三维理论模型构想的研究中, 虽肯 定了 “科研能力” 的重要性, 但是, 同样忽视了教育 科学研究素养作为整体在教师专业素质结构中的核 心地位。鉴于此, 我们认为, 应在教师素质结构中加 入教育科学研究素养维度, 使其形成一个四维金字塔 结构的理论模型。构想如图 3 所示:

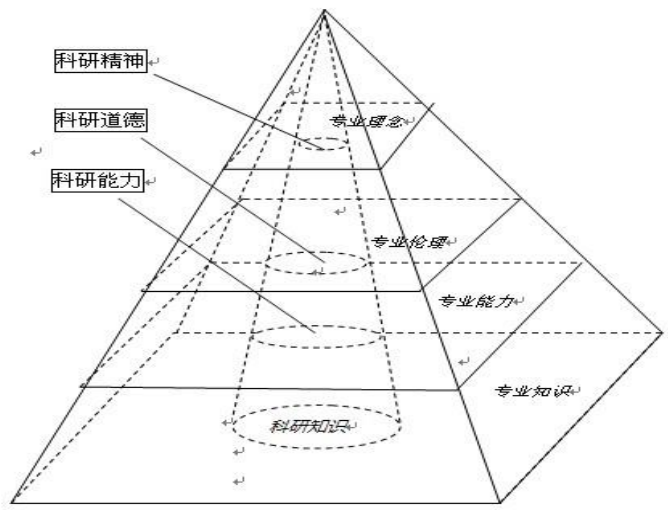

图 3: 教育科学研究在教师专业发展中的核心动力模 型

如图所示, 在以往研究基础上, 可以把教师专业 素质的结构体系划分为教育科学研究素养、专业理 念、专业知识、专业能力和专业伦理五个方面。其中, 教育科学研究是教师不断提升自身专业水平的“能量 棒” ; 是教师专业素质结构的内核, 居于中心位置。 它通过系统间的重叠和交叉影响着其他系统的工作 状态和工作质量。而教师的专业知识、专业能力、专 业伦理和专业理念由底层逐渐向高层分居于金字塔 的不同部位, 共同构造教师专业基础的平稳底座和主 体结构。专业理念是 “教师在对教育专业本质理解基 础上形成的关于教育的观念和理性信念, 是统率教育 活动总的思想意向”。 ${ }^{[11]}$ 因此居于金字塔顶端。专业 知识和专业能力是教师顺利进行教育教学活动的基 本要求, 二者共同构成了教师专业素质的底线, 因而 居于金字塔的底部。教师的专业伦理主要指教师从事 教育教学活动时所坚持的专业道德操守、专业信念、 专业情感, 以及遵守的专业行为规范。它是教师从单 纯地从事教育教学活动上升为享受专业生活的推进 剂。教师专业素质的五个子系统相互作用和影响, 构 成了完整的教师专业素质结构体系。对科研素养本身
来说, 则包含科研知识、科研能力、科研道德以及科 研精神等子系统。鉴于以往的研究已对上述教师专业 素养的内涵和构成做过较多研究, 本文不再赘述。

\section{4. 结论}

总之, 教育科学研究素养应该成为教师专业素质 结构的内核, 教育科学研究应是教师专业发展的核心 动力, 而非教师专业能力维度的一个组成部分。这对 于探讨教师专业素质结构问题, 以及提升教师专业化 发展等具有不可忽视的重要意义和实际价值。

\section{REFERENCES}

[1] Gu Mingyuan. The dictionary of Education (Volume 2) $[\mathrm{m}]$. Shanghai: Shanghai Educational Science Press, 1990.16

[2] Zhang Hongxia. Difficulties and breakthroughs in Teachers' professional quality evaluation [J]. Education review, 2012 (4). 60

[3] Xie Anbang, Zhu Yubo. On the category and structure of teachers' quality [J]. Research on teacher education, 2007 (3). 2

[4] Li Jianhui, Wang Jingjing. New Exploration on the structure of teachers' professional quality [J]. Contemporary teacher education, 2010 (3). 11

[5] Li Jianhui, Wang Jingjing. New Exploration on the structure of teachers' professional quality [J]. Contemporary teacher education, 2010 (3)

[6] Yuan Zhenguo. Educational research methods [M]. Beijing: Higher Education Press, 2006.4.1

[7] Xie Anbang, Zhu Yubo. On the category and structure of teachers' quality [J]. Research on teacher education, 2007 (3). 4

[8] Hanyanping. The quality of postgraduate scientific research and self-cultivation [d]. Guilin: Guangxi Normal University, December 2012

[9] Li Jianhui, Wang Jingjing. New Exploration on the structure of teachers' professional quality [J]. Contemporary teacher education, 2010 (3), 12-13

[10] Su Zhigang. On the three dimensional structure of teachers' professional quality in Higher Vocational Colleges [J]. China higher education research, 2013 (12), 75

[11] Li Jianhui, Wang Jingjing. New Exploration on the structure of teachers' professional quality [J]. Contemporary teacher education, 2010 (3), 13. 